\title{
Enterprise Application Integration - The Cloud Perspective
}

\author{
Jörg Lässig and Markus Ullrich \\ University of Applied Sciences Zittau/Görlitz \\ Department of Electronical Engineering and Computer Science \\ Brückenstr. 1, 02826 Görlitz, Germany \\ \{jlaessig, mullrich\}@hszg.de \\ http://f-ei.hszg.de/ead
}

\begin{abstract}
So far, asynchronous messaging has proven to be the best strategy for enterprise application integration (EAI) success. However, building and deploying messaging solutions causes several problems for developers and new technologies and computing paradigms as cloud computing demand for new solutions. There are more than sixty enterprise integration patterns that are designed to effectively develop messaging solutions for enterprises. The tutorial introduces the visual notation framework to describe large-scale integration solutions across different systems and technologies. This includes examples covering a variety of different integration styles and techniques. In a case study we illustrate the application of the patterns in practice and review existing and emerging standards. Also we try to shed light into the future of EAI. In particular cloud integration is an upcoming trend which is discussed in the tutorial, addressing advantages and limitations of this and other modern EAI strategies and architectures. Looking at open-source solutions for enterprise service buses and messaging systems, we also provide practical advice on designing code that connects an application to a messaging system. This provides information to help the practitioner to design EAI or cloud integration solutions by applying the introduced knowledge.
\end{abstract}

Keywords: Enterprise application integration, cloud integration, integration styles, enterprise service bus, open-source ESB systems, integration patterns, messaging, business process integration.

\section{Introduction}

Nowadays requirements for software systems in enterprises are changing frequently due to changes on the business side but also due to emerging new technologies such as significant progress in the cloud computing field. Also, requirements and applied technologies usually differ between enterprises - even in the same industrial sector- significantly. This is due to the availability of various solutions for similar problems, differences in the historic development of an enterprise, different management styles, different business strategies and goals, different levels of process automation and management, etc.

F. Daniel, P. Dolog, and Q. Li (Eds.): ICWE 2013, LNCS 7977, pp. 522-525, 2013.

(C) Springer-Verlag Berlin Heidelberg 2013 
Thus, it is impossible to offer all-in-one software solutions to satisfy all the needs of every enterprise. The obvious practical approach is of course to have different software tools for special purposes and to choose them accordingly from a selection of available systems. In the usual case, the choice is feature-based and hence often systems from different software vendors are applied to different needs within the same enterprise. The need of communication between those systems was/ is often not needed initially but is becoming increasingly important in a globalized environment and in times of the introduction of new paradigms as Industry 4.0 [6] or information partnerships between companies.

\subsection{Enterprise Application Integration}

According to the above described setting, the selected systems of a company often do not support appropriate interfaces to communicate with each other directly. Also, the coupling of several systems in a pairwise manner is inefficient. This is where enterprise application integration (EAI) comes into play: The need to exchange information between those systems has to be satisfied by a robust and flexible enterprise application integration strategy. According to Gartner there are four major data delivery styles 1 . A frequently used and effective solution for EAI is messaging since it offers the same loose coupling between applications as file transfer and -at the same time- it is almost as consistent and reliable as a shared database but with no obvious performance bottleneck. Despite those advantageous characteristics, the asynchronous nature of messaging as well as integrating software systems with a variety of different interfaces can be a significant challenge for first time developers of a messaging system.

\subsection{Cloud Integration}

Currently, the cloud paradigm influences various enterprise IT solutions [7] and the same is true for the development of state of the art EAI solutions [1]. Cloud computing solutions in enterprises also trigger a demand for new integration solutions, e. g., SaaS-to-SaaS (S2S) integration, Cloud-to-Enterprise integration $(\mathrm{C} 2 \mathrm{E})$ or even cloud business process management solutions (Cloud-BPM)2.

To formalize different approaches, enterprise integration patterns [2] can help to reduce the complexity of integration solutions in a standardized way. Here, basic messaging patterns are considered and the tutorial highlights in which situation which pattern should be applied. This also includes an review of opensource enterprise service bus (ESB) solutions that support messaging, the application of EAI patterns for common integration scenarios and discussions on how new challenges and technologies can be integrated.

\footnotetext{
${ }^{1}$ E. Thoo, T. Friedman, M. A. Beyer. Critical Capabilities for Data Integration Tools: Common Data Delivery Styles.

2 G. Johnson. Cloud Integration Defined. Can Cloud BPM Be Far Behind?
} 


\section{Tutorial Synopsis}

After outlining the need of EAI in modern enterprises to be competitive, we discuss the most frequent challenges when applying EAI strategies in practical settings, such as the integration of critical business functions in the integration scenario or a lack of control concerning participating applications. Typical integration settings range from the integration of a few applications in a single company for special needs up to the integration of various systems and processes in supply chains and enterprise networks or dynamic environments such as virtual enterprises as introduced in [3/4/5].

The attempt to reduce the complexity of integration tasks motivates the development of standards to handle and manage those various flavors of integration scenarios. We feature the common visual notation for integration solutions as introduced by Hohpe and Woolf in [2] so that integration scenarios can be defined and described consistently and high-level.

Next we discuss basic integration styles like file transfer, relational databases and remote procedure calls with a special focus on asynchronous systems and message processing. Here, the topics range from the construction of messages over the characteristics of messaging channels, routing and transformation patterns and also endpoints. Even patterns for managing messaging systems are considered. Additionally, we describe architectural styles and patterns like pipes and filters or message brokers.

Subsequently, we also give an introduction to EAI in the cloud while explaining the different characteristics and levels of cloud integration and the additional challenges of application integration compared to data integration in the cloud. This includes also security considerations in this open and vulnerable environment. Compared to traditional environments new security models across clouds are needed.

On this firm conceptual basis, we review current technologies and standards for EAI solutions and also point out further standardization needs. As one way to implement integration patterns, web services are examined. ESBs are used for the integration of complex and heterogenous EAI scenarios and can be seen as a general approach to implement service-oriented architectures efficiently. There is a high number of different ESB systems available. Focusing further on the implementation side, we showcase some of the most popular open-source ESB solutions for EAI in a live demo and compare them in terms of features, e. g., supported patterns, performance, documentation, usability, maturity or the support of work flows and work flow engines. Typical is, e. g., the support of the WS-Business Processes Execution Language (BPEL) to implement work flows which orchestrate web services.

In a case study we implement an non-trivial exemplary integration scenario and highlight the most important considerations while designing an effective messaging solution, which illustrates certain patterns in a practical setting. The tutorial is concluded with an outlook on emerging trends and future directions of EAI. 


\section{Bibliographical Sketch}

Jörg Lässig is a Full Professor in the field of Enterprise Application Development at the Department of Electrical Engineering and Computer Science at the University of Applied Sciences Zittau/ Görlitz since 2011. He holds degrees in Computer Science and Computational Physics and received a Ph.D. in Computer Science for his research on efficient algorithms and models for the generation and control of cooperation networks at Chemnitz University of Technology, which he finished in 2009. Afterwards he has been participating in various research projects at the International Computer Science Institute at Berkeley, California and at the Universit della Svizzera italiana in Lugano, Switzerland. He is currently focusing on various topics in the context of sustainable information technologies and applications which includes the directions sustainability in enterprise IT, green information systems, logistics and supply and business intelligence.

Markus Ullrich is currently a research associate at the University of Applied Sciences Zittau/Görlitz where he received his M.S. and B.S. in Computer Science in 2010 and 2012, respectively. From 2009 to 2012, he worked as a software developer at the Decision Optimization GmbH where he developed and tested data mining algorithms for predictive maintenance. His current research is focused on cloud computing, cloud integration, distributed systems and privacy preserving data mining.

\section{References}

1. Erbes, J., Motahari Nezhad, H.R., Graupner, S.: The future of enterprise it in the cloud. Computer 45(5), 66-72 (2012)

2. Hohpe, G., Woolf, B.: Enterprise integration patterns: Designing, building, and deploying messaging solutions. Addison-Wesley Professional (2004)

3. Lässig, J.: Algorithms and Models for the Generation and Control of Competence Networks. Mensch und Buch Verlag (2009)

4. Lässig, J., Heinrich, S., Dürr, H.: Intelligent support system for enterprise cooperation management. In: Proceedings of the 4th Indian International Conference on Artificial Intelligence, Bangalore, India, pp. 1626-1645 (December 2009)

5. Lässig, J., Trommler, U.: New approaches to enterprise cooperation generation and management. In: Proceedings of the 12th International Conference on Enterprise Information Systems (ICEIS 2010), Funchal, Madeira, Portugal, pp. 350-359 (2010)

6. Meisen, T., Meisen, P., Schilberg, D., Jeschke, S.: Digitale produktion via enterprise application integration. In: Automation, Communication and Cybernetics in Science and Engineering 2009/2010, pp. 609-622. Springer (2011)

7. Ullrich, M., ten Hagen, K., Lässig, J.: Public cloud extension for desktop applications-case study of a data mining solution. In: 2012 Second Symposium on Network Cloud Computing and Applications (NCCA), pp. 53-64. IEEE (2012) 